\section{Advancing treatment of metastatic cancers: from research to communication - where do we need to go?}

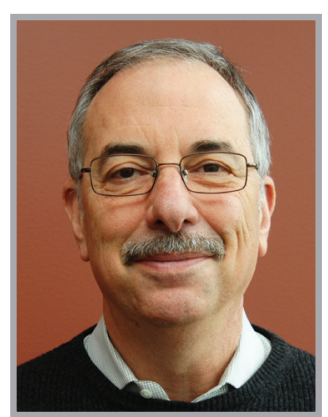

Bruce Zetter* speaks to Francesca Lake, Managing Commissioning Editor: Bruce Zetter is the Charles Nowiszewski Professor of Cancer Biology at Harvard Medical School in Boston (MA, USA). Dr Zetter received a BA degree in anthropology from Brandeis University in Waltham (MA, USA) and a PhD from the University of Rhode Island in Kingston (RI, USA). He completed fellowships at Massachusetts Institute of Technology in Cambridge (MA, USA) and at the Salk Institute in San Diego (CA, USA). Subsequently, he was an assistant research biochemist at the University of California in San Francisco (CA, USA) before joining the faculty at Harvard Medical School. At Harvard, he directed the course in human physiology taken by all medical students. He further served as the Chief Scientific Officer at Boston Children's Hospital (MA, USA), where he directed the research efforts for the hospital. Dr Zetter has made major discoveries on the mechanisms underlying tumor metastasis and on the detection and treatment of late-stage tumors. As an internationally recognized expert in the field of tumor metastasis, Dr Zetter has chaired multiple international research conferences and grant review panels for agencies such as the US $\mathrm{NIH}$ and the US Department of Defense. He also chaired the NASA committee that selects scientific projects for the space shuttle. Dr Zetter has a strong interest in the interactions of academic and corporate institutions and has served as an advisor to more than 30 biotechnology and pharmaceutical companies, as well as to venture firms and investment companies.

\section{Q What sparked your interest in cancer research?}

Initially, in 1970, I was a graduate student in microbiology. In 1971, Richard Nixon the then President of the USA - declared a war on cancer and dedicated funds to fight the disease. That fight is now more than 40 years old, and I have been participating in it since then.

I finished my degree in microbiology and then did a postdoctoral fellowship with John M (Jack) Buchanan at Massachusetts Institute of Technology (MA, USA). His laboratory was becoming interested in cancer, and I wanted to do research that was disease-relevant and that made an impact on human health. Therefore, I switched from studying bacteria to studying cancer cells, which I have been studying ever since.

*Boston Children's Hospital, Boston, MA, USA and Harvard Medical School, 300 Longwood Avenue, Boston, MA 02115, USA; bruce.zetter@childrens.harvard.edu

\section{KEYWORDS:}

- academic/corporate interactions - cancer • metastasis • teaching and mentoring 
Q What led you to your current research in cancer metastases?

I have navigated several different interests over time. Early in my career, I was interested in the factors that stimulate the growth of tumor cells. At that time, we thought that only the tumor cell was important, that the cells around it didn't make any difference and that a tumor was actually just a sphere of tumor cells. Now we know there is so much more to the tumor than just the tumor cells.

Then, in 1978, I came to the Boston Children's Hospital (MA, USA) to work with Judah Folkman, who had developed the theory of tumor angiogenesis. He reasoned that blood vessels were critically important in supporting the growth of the tumor, and that attacking tumor blood vessels could retard tumor growth by restricting their nutrient supply. I worked directly with Folkman, an amazing scientist and mentor, for a few years and then developed my own interest in tumor metastasis. Subsequently, since the mid-1980s, I have been working primarily on how metastases form and how we can detect and treat them.

Today, I am much more interested in treating tumors once they have spread, as $90 \%$ of all cancer deaths result directly from metastases. Our current research is really threefold. One aspect is determining the mechanisms by which tumors grow in the secondary site - we are not so interested in how they get there - so we can stop them from taking over important organs, such as the brain, lungs, liver and bone marrow. Second, we have a biomarker component, which again is focused on the metastases in terms of taking samples from patients at the time of diagnosis and determining if we can predict their future outcome. The question is, are they likely to have undetectable metastases that are already present at diagnosis, which will lead to large, life-threatening metastases later on? We are therefore trying to predict this from patient urine samples taken at the time of diagnosis. The final goal we are attempting is to develop new therapies that specifically treat late-stage metastases wherever they are located. We have evidence that metastatic tumors are sufficiently different from the primary tumors that the drugs that will treat them effectively will be unique; they will not be modeled by studying the primary tumor. Therefore, we are working to discover drugs that selectively target and inhibit aggressive cells in the disseminated metastases. We are targeting the cells that cause cancer mortality.

\section{Q What do you feel is the biggest challenge} facing the field today?

There are dozens of challenges facing the field today. I think the field, in terms of my tenure of being in cancer research, has really accelerated progress in the last few years with more new treatments, modalities and ways of seeing the tumor as a total organism. We can pick out the various components of the tumor and treat each part with different types of treatment, be they cytotoxics or immune therapy, or antiangiogenic therapy or anti-inflammatory therapy. Thus, I think that substantial progress has been made in the laboratory, but now we have to conduct clinical trials to determine how to harness all of these new approaches. This is going to become very difficult. We will need to combine these treatments, either simultaneously or in series, to find a rational way to determine the best treatment algorithms. I believe that trying to harness the huge amount of information that we have now and the large number of new drugs that are working their way through the pipeline into effective treatments, in combination or in series, is the biggest challenge facing the cancer community today. We also need to ensure that each patient is getting the appropriate treatment for his or her own tumor, not some generic protocol, and this is becoming more and more possible.

The second biggest challenge is sufficiently educating the clinical population as new treatments, regimens and combinations are constantly emerging, yet sometimes the last people to hear about them are the physicians treating the patients. How can we better communicate what is working and what is likely to be working in what context, so physicians in the most remote areas have access to the newest kinds of treatments?

Q How would you suggest we address this challenge in communication?

Communication will come from networks, whether they be government, large groups of physicians or organizations. However, the information must be rapidly disseminated and targeted; it can't just be put up on the web with 'when you have time, click here.' It has to be dispersed in a targeted way so that the most important information reaches the people who are executing the treatments. 
Q What do you feel is the biggest achievement of your career so far?

I think one hopes to have a few good achievements and that the totality of one's career makes a difference and occasionally changes minds in the field. Very early in my career, when I was working on angiogenesis, people thought that the most important component of this was the growth of the endothelial cell in the blood vessel; however, our work showed that the migration of endothelial cells toward the tumor was a critical component that preceded the proliferation. Now people talk about the different types of cells - tip cells and stalk cells - in the blood vessels as they are moving, but I think our work was first to show that there were multiple components to the angiogenic process, not just blood vessel cells growing in a mass.

I am also hoping that the work we are doing at the moment will have important implications for cancer therapy. This latest work is on finding drugs that specifically target the most aggressive tumors when they are in the secondary site and the patient is near death, because those are the hardest things to study. A lot of historical research in cancer biology has been to inject tumor cells into the back of a mouse and then start to treat the with your drug of interest on the next day and then see if you can make that primary tumor grow more slowly. Yet, if you ever develop a drug through that mechanism and get it into the clinic, you find that the patients you are going to be treating have widespread metastatic disease and have failed several other treatments. They are so far removed from a mouse with one new tumor on its back that the information from that experiment is of almost no value. We now have developed better animal models of human metastatic disease, and our group and others are treating animals only after they have widespread metastases in order to try to find drugs that are specific for that situation. We look forward to the day, hopefully soon, when cancer patients can be expected to live longer lives, even when they have very aggressive disease because of new drugs that specifically target late-stage, metastatic cancers.

Q You have been recipient of three different teaching awards from the students from Harvard Medical School for excellence as a teacher \& as a course director. Could you tell us how you became involved in education, what you enjoy about it \& what you think the benefits are?

Blame my parents! They firmly believed in the importance of oral communication and public speaking and I was encouraged to take public speaking classes from the seventh grade onwards. I am very comfortable communicating and I love to communicate in any kind of educational setting, whether it is to undergraduates, graduate students, medical students, scientists at a lecture or a lay audience. I think it is an important part of what we do as scientists, to be able to communicate both in the written word and orally. I think that too few people who go into science are given sufficient writing and speaking training, so, for me, it was just natural, having been trained from a very early age to deliver talks and speeches, among other things. It was natural to start to be an educator and I always enjoyed trying to find interesting or novel ways to explain things to students or other kinds of audiences. Therefore, I think it comes from childhood guidance indicating that communication is really important.

\section{Q Are you still involved in teaching?}

I taught for approximately 20 years on our physiology course at Harvard Medical School and directed that course for approximately 10 years. I now give fewer course lectures, but I do more lecturing to larger audiences, whether it is at a scientific conference or to a lay audience. I think we have an obligation to be able to communicate our work to what should be its major audience - the public.

\section{Q Do you think anything needs to be}

changed with regard to how we communicate to the public?

It is an important question. We have to first make sure that people visit their doctors throughout their lives. Most young adults never see a doctor unless they are sick, so we don't have enough baseline data on their normal state. We also have to deemphasize the reporting of individual experiments that may not bear fruit in the clinic for a very long time and do a better job of summarizing the trends in cancer research that have made a real difference in extending patient lives. We don't always do a good enough job of sharing the real changes and successes in cancer treatment, as well as the continuing challenges that we face. 
Q You also have served as Vice President of Research \& Chief Scientific Officer at the Boston Children's Hospital, as well as on the scientific advisory boards of several biotechnology \& pharmaceutical companies. Could you tell us about these roles $\&$ how you balance your time?

I was happily running my research laboratory when our Vice President of Research took a different job and the President of the hospital called me in and said, "How would you like to be Vice President of Research Administration?" and I said, "What is a Vice President and what is research administration?" Research administration, which is an enormously important job, is making sure that the grants office, patents office and financial office are all well run, but it was not something that I was particularly trained in. Then we decided to create a new job that encompassed all of research - not just administration and compliance, but also strategy and mentoring and advocacy for faculty. First we called it Vice President for Research and later, Chief Scientific Officer. Over the years that I performed those jobs, I came to feel that the most important aspect was the advocacy role. Setting strategy was critically important, but it was also critical to serve as an advocate for the scientists, especially because we are in a hospital, where scientists need particular protection and support for their mission. Some of the scientists are $\mathrm{MD} / \mathrm{PhDs}$, some are $\mathrm{PhDs}$ and some are $\mathrm{MDs}$, so it doesn't matter what kind of training they have, they need protection and advocacy, especially the physician scientists who are being asked to do two different full-time jobs and do them both well. In addition, this involved advocacy for the institution to make sure that we were exposed to the right kinds of federal agencies and interactions with companies. It also involved some fundraising with the hospital development team, because when you are responsible for research, you have to be able to communicate to people who may want to make donations to support high-risk, innovative science. While I was doing that, I was also running a research laboratory and doing some outside activities with biotech companies. At the time, when people asked, "What percentage of your time were you devoting to each job?” I would say, "100\% to research, $100 \%$ to administration, approximately $50 \%$ to outside activities and the rest to family." That is not sustainable over the long term, so I gave up the administrative role after 9 years, and what I do now is primarily research, but also advising and consulting to biotech companies, which is something that started very early in my career.

When I was a postdoctoral fellow, a friend started a biotech company and asked if I would like to be an advisor. Since then, I have been an advisor or scientific advisory board member for more than 30 biotech companies. Those jobs don't last too long because a start-up company either goes out of business or becomes a mature company, and as they become something else they need different kinds of advisors. Thus, a typical tenure working for one company might be 3 or 4 years, and then new ones will start up. I haven't done quite so much with large pharmaceutical companies, although some friends advise those companies more; I found that my niche is in helping brand new companies get started and find their way and in really trying to focus on developing their primary products.

One thing I have noticed is that when scientists start companies, they often want to run them like research laboratories and do lots of experiments to get to more theoretical understanding, but eventually they have to have a product. They only have so much money, so one of the things I try to help companies do is focus on their major product pipeline and make sure we get those products out of the laboratory and into the marketplace. That has been a fun part for me, because, I think, importantly for scientists, especially those working in clinically applicable science, you have to figure out how to get your research discoveries to patients. That requires patenting, trying to secure licensing by a larger company or, if that is not possible, starting a company around your discoveries, raising money and then making sure that company succeeds at least long enough to get the drugs, devices or diagnostics into the clinic. Sometimes there is a natural evolution, or maybe it is an ecological system, where companies start. Many go under, but their technology or the company itself is bought by another company that may have different priorities. Even if for one reason or another, the small biotech company doesn't succeed or is bought early, the good technologies often make it to the clinic. I have seen promising technologies pass through three different companies before they reach the clinic.

Another issue regarding academic investigators is that they sometimes don't realize the importance of patenting their discoveries. This is not taught often enough in graduate schools. 
If a new drug, new test or new method is never patented by the discoverer or inventor, then they will never get to the clinic because no company will take something without a patent. Again, one of my jobs when I was in administration and, even now, is trying to educate young scientists on how to protect their work and ensure that it is going to be something that will be licensed and get to a company and to the marketplace. We have to keep in mind that the patient is our most important client.

Q You are also an expert medical witness in court cases involving health \& biotechnology. Could you briefly tell us about this role?

This is really interesting and all kinds of different cases come to court, but let's just talk about patent cases, as they are one of the most common. As a new drug or device becomes successful, especially if it is financially successful, other companies will want to make their own version. This frequently generates a court challenge to the initial patents. You will find that there are many ways to try to circumvent a patent. When you look at both sides of a patent dispute, you often find a large gray area; it isn't black and white. Very similar to high school debating, you can see both sides, yet you have to believe in the side you are taking and you have to cut to the essence and try to make the most important points and do so in a contentious setting. There are often two phases: a writing phase when you are doing research, responding to questions and writing briefs; and the court phase, which can be very challenging and, sometimes, stressful. Many people do it once and never again, but there are a few people who are good at it or enjoy it and continue doing it. I found it stressful but also satisfying and enjoyable to do, especially when you feel strongly about something and believe that what you are doing is really going to help the patients. There are definitely some stresses involved when you are being cross-examined but, as scientists, we are used to hard questions.

\section{Q What do you think are the current hot topics in cancer research?}

The role of the microenvironment, the ancillary cells in the tumor, is something we didn't always know about and has become exceptionally important to our understanding of cancer. Folkman's work on blood vessels in tumors was an early example of this kind of research. We know now that the blood vessels, inflammatory cells and connective tissue cells in a tumor, even the bacteria in the gut, are hugely important for the patient's outcome. However, what we don't yet understand is how that system works as a whole. We have some people studying the neutrophils, others studying the macrophages, others studying the endothelial cells, others studying the fibroblasts and some studying bone marrow stem cells that travel to the tumor, and yet we need a more systematic approach to understand the tumor as a whole organism. The question here is, what is going on in the tumor and what components of that tumor plus its microenvironment do we have to fight in order to combat the whole tumor?

The other topic that is emerging is the topic of cancer dormancy. There are probably very few cancer patients diagnosed who don't have some cancer cells that are already disseminated. Now, for some people, those cancer cells will sit in a dormant state forever and, for others, some time after removal of the primary tumor, the dormant metastases will start to grow and take over the organ. We now understand that those cells are present in many cancer patients, and we know that there are going to be triggers that cause them to leave dormancy and become aggressive; however, we don't know what those triggers are or how to delay that process, so I think the notion of trying to keep metastases dormant is something that actually might be easier than trying to break large scale metastases. This is a kind of prevention, but it isn't prevention from getting cancer; it is prevention from having your micrometastases explode.

\section{Q What progress do you think will be made} to treatment in the next 10 years?

What I see are really combinations of different modes of treatment. We will have combinations of drugs that are similar, so you could imagine, let's say, that the tumor has mutations that promote cell growth. You might want to treat with inhibitors to all three of those proteins. This, though, would only treat one aspect of the tumor and may not be effective. At the same time, however, you might want to treat with one or more antiangiogenic drugs, one or more anti-inflammatory drugs and something that is going to stimulate a specific immune response. Just recently, the field of tumor immunology has come back to the forefront of cancer research. The excitement here is the possibility of very long-term responses in those who benefit from 
immunotherapy. My feeling is that someone who is diagnosed with cancer in 10 years is going to get a well-orchestrated regimen over time of a variety of modes of cancer treatments, and this will certainly lead to longer lives for cancer patients.

Q Finally, do you have any other thoughts for our readers?

We haven't touched on mentoring. I think that scientists really rely on mentors. You rely on mentors for teaching you how they do science. There are many ways to be a successful mentor. Principally, you simply have to care about the lives and careers of those you work with. Seeing how someone who is a successful mentor operates, determining what you want to emulate, and then seeing what mistakes they have made and what mistakes you don't want to repeat will make you a better leader and mentor in the future. Having someone shepherd your career is a terrific advantage. I believe the public sometimes thinks that academics live in an ivory tower that is isolated from political intrigue. Like any other career, there is political intrigue in science and there are ways to protect and promote other people's careers. You need someone to be looking after you in that way, and I have been really fortunate to have some terrific mentors: Paul Cohen, who was my graduate advisor when I was a PhD student working in microbiology, Jack Buchanan at Massachusetts Institute of Technology and Judah Folkman at Boston Children's Hospital, a brilliant physician scientist who paved the way to allow me to be successful in science. It is incredibly important for young scientists to find a few really good mentors, cultivate them, spend time with them, figure out what they do well and not well and impart a feeling of the excitement of science.

\section{Disclaimer}

The opinions expressed in this interview are those of the interviewee and do not necessarily reflect the views of Future Medicine Ltd.

Financial \& competing interests disclosure

$B$ Zetter currently serves on the scientific advisory boards of the following companies for which he receives financial consideration in the form of income or stock in the companies: Blend Therapeutics, Cerulean Pharma and SynDevRx. $B$ Zetter has no other relevant affliations or financial involvement with any organization or entity with a financial interest in or financial conflict with the subject matter or materials discussed in the manuscript apart from those disclosed.

No writing assistance was utilized in the production of this manuscript. 